\title{
Search for exotic matter from gravitational microlensing observations of stars
}

\author{
M.B.Bogdanov ${ }^{1}$ • A.M.Cherepashchuk ${ }^{2}$
}

(C) Springer-Verlag $\bullet \bullet \bullet$

\begin{abstract}
We consider small-scale spheroidal clusters of weakly interacting massive particles in our Galaxy as noncompact gravitational microlenses and predict the appearance of caustics in the plane of a lensed source. The crossing of these caustics by a lensed star can produce a large variety of light curves, including some observed in actual microlensing events that have been interpreted as manifestations of binary gravitational lenses. We consider also observable effects during the gravitational microlensing of stars of non-zero angular size with a given brightness distribution across their disks by such an exotic objects as natural wormholes and objects whose space-time environment is described with the NUT metric. We demonstrate that, under certain conditions, the microlensing light curves, chromatic and polarizational effects due to the properties of the lens and the star disk brightness distributions can differ considerably from those observed for a Schwarzschild gravitational lens, so that their analysis can facilitate the identification of such objects.
\end{abstract}

Keywords gravitational microlenses; dark matter; WIMP; wormholes; NUT metric;

\section{Introduction}

The idea of detecting dark matter via its gravitational action on the light arriving from distant stars suggested by Byalko (1969) and Paczynski (1986) was realized

M.B.Bogdanov

Chernyshevsky State University, ul. Astrakhanskaya 83, Saratov, 410012 Russia

A.M.Cherepashchuk

Sternberg Astronomical Institute, Universitetskii pr. 13, Moscow, 119992 Russia in observations of gravitational microlensing of stars by massive compact halo objects in our Galaxy (Alcock et al. 1993). Various international teams - MACHO, OGLE, PLANET, and others - have detected several hundred microlensing events for stars in the Magellanic Clouds and in the bulge of the Milky Way. However, the nature of the objects acting as gravitational lenses remains unclear. According to the statistics of observations by the MACHO team (Alcock et al. 2000), the lens masses are in the stellar-mass range. In two cases, the masses exceed the limit for a neutron star, while the luminosities of these lenses are no higher than that of the Sun (Bennett et al. 2002); these objects are probably black holes.

A considerable fraction of the lenses may be dwarf stars in our Galaxy. This is favored by statistical arguments Komberg. Kompaneets, \& Lukash 1995; Kerins 1997), and also by the analvsis of light curves (Bogdanov \& Cherepashchuk 1998; Gaudi et al. 2002). In two cases red dwarfs, that created the effect of gravitational lens, were directly observed by the Hubble Space Telescope (Alcock et al. 2001; Kozlowski et al. 2007). As noted in the recent review of Kochanek, Schneider, \& Wambsganss (2006), the fraction of lenses from the dark matter can be sufficiently small. However, uncertainties in the properties of the gravitational lenses still admit a large variety objects of stellar masses as candidates for this role.

Along with the black holes mentioned above, the possibilities of the lenses being small-scale clusters of weakly interacting massive particles (WIMPs) (Gurevich. Zvbin. \& Sirota 1997: Sazhin, Yagola, \& Yakubov 1996; Zakharov 1999; Bogdanov 2001) have also been considered. Lenses of more exotic types of matter have also been suggested, in particular, tunnels in space-time (wormholes) (Eiroa. Romero. \& Torres 2001: Safonova, Torres, \& Romero 2002; Bogdanov \& Cherepashchuk 2002), as is predicted by general relativity. The papers 
Rahvar \& Nouri-Zonoz (2003) and Rahvar \& Habibi (2004) considered the gravitational microlensing of point sources by hypothetical objects whose local regions of space-time are described with the NUT metric, and compared theoretical light curves to observations.

The aim of the current paper is to study effects observed for gravitational microlensing of stars by the exotic objects. It is shown, in particular, that observations of stellar microlensing evens can reveal fine photometric, chromatic, and polarizational effects due to properties of the lens and lensed star: its angular size, the brightness and polarization distributions across the stellar disk, and the wavelength dependences of these characteristics. Though the manifestations of such effects are fairly small, their analysis can contribute to identification of the lens type.

\section{Microlensing by Clusters of Weakly Interacting Massive Particles}

One approach to the solution of the dark-matter problem supposes the existence of weakly interacting massive particles (WIMPs) that formed at an early stage of the evolution of the Universe. Because of their gravitational interaction, these particles should have produced a hierarchical structure of clusters with certain scaling properties. The masses of the smallest objects are of the order of a solar mass. Gurevich. Zybin. \& Sirota (1997) fitted the mass density of the WIMPs in a smallscale object with the following function of the distance $r$ from the center of the cluster:

$$
\rho(r)=\left\{\begin{array}{cc}
\rho_{0}, & 0 \leq r \leq r_{c} \\
\rho_{0}\left(r / r_{c}\right)^{\alpha}, & r_{c} \leq r \leq R_{x} \\
0, & r>R_{x}
\end{array}\right.
$$

The exponent in (1) is also a scaling parameter, equal to $\alpha=1.8$. The radius of the core, which has a constant density $\rho_{0}$, is denoted $r_{c}$ and can range from 0.05 to 0.10 of the cluster radius $R_{r}$. According to Gurevich, Zybin, \& Sirota (1997), a typical cluster has a mass of $M_{x}=0.1-1.0 M_{\odot}$ and a radius of $R_{x} \sim 10^{14}-10^{15}$ $\mathrm{cm}$.

The particles interact weakly both with each other and with external matter, so that the detection of such clusters seems verv unlikelv. However. Gurevich \& Zybin (1995) pointed out that, if such objects are located along the line of sight connecting an observer and a normal star, they can deflect the light rays from the star; in view of the masses of the clusters involved, this can be considered gravitational microlensing. Gurevich, Zybin, \& Sirota (1997) computed flux-variation curves for the gravitational microlensing of stars by non-compact lenses made up of the WIMP clusters. The shapes of these curves can be rather similar to those of a Schwarzschild lens, with a symmetric profile and a sharp maximum, albeit with broader wings. In their comparison of these curves with the first observational data obtained by the OGLE group. Sazhin, Yagola, \& Yakubov (1996) showed that in two of eight cases the observed light curves can be better described by a non-compact lens model. Later, however, it was shown that the broadening of the wings of these light curves can also be explained in a simpler way - by either blending (Wozniak \& Paczynski 1997) or by the radiation of the lens itself, if it is a normal star (Bogdanov \& Cherepashchuk 1998).

Zakharov (1999) analyzed a spherically symmetric nonsingular model for a non-compact gravitational microlens, which describes the expected density distribution in a WIMP cluster better than earlier models. Under certain conditions, such a microlens can have circular caustic curve in the plane of a lensed source and produce a symmetric light curve with two maxima and a dip between them. Similar curves can also be produced by binary gravitational lenses and have already been observed in some microlensing events.

In reality, the spherical symmetry of the density distribution in a non-compact object can break down. One factor that can lead to such a disruption of the symmetry is the tidal deformation of WIMP clusters as a result of their gravitational interactions with each other or with stars of the Galaxy. The possibility of such deformations was pointed out by Gurevich, Zybin, \& Sirota (1997). However, the probability of a close encounter involving appreciable tidal forces is very low. Another factor - the oblateness of the particle cluster by rotation - seems more likely.

Rotation is a general property of systems that form as a result of the gravitational contraction of matter. Rotation explains, for example, the observed oblate shapes of elliptical galaxies and globular star clusters. In the origin of rotation in such systems, an important role should be played by gas-dynamical effects, which are obviously absent in the case of weakly interacting particles. There is, however, a mechanism capable of inducing rotation of the particles exclusively via gravitational interactions. This mechanism, first suggested by Hoyle (1949) and then developed in more detail by Peebles (1969), is based on the mutual attraction of tidally deformed clusters. In this case, the orbital momentum associated with the relative motion of the clusters is transformed into rotational momentum of each of the interacting clusters. In the early Universe, when WIMP clusters formed, their spatial density was substantially higher than now. Close encounters occurred 
rather frequently, and the Hoyle - Peebles mechanism could be fairly efficient.

The observed manifestations of a transparent gravitational lens depend on the distribution of the surface mass density $\Sigma(\xi)$, where $\xi$ is a vector defining the position of a point in the plane of the lens. Here and below, we write vector names in bold font. For the spherically symmetric law (1) in a coordinate system whose origin coincides with the projection of the center of the particle cluster onto the plane of the lens, $\Sigma(\xi)$ is given by the integral

$$
\Sigma(\xi)=2 \int_{0}^{\sqrt{R_{x}^{2}-\xi^{2}}} \rho\left(\sqrt{\xi^{2}+\mathbf{z}^{2}}\right) d z .
$$

It is obvious that the distribution of the surface mass density in this case is rotationally symmetric. Further, we consider the dimensionless density

$$
\kappa(\xi)=\Sigma(\xi) / \Sigma_{c r},
$$

where the so-called critical density $\Sigma_{c r}$ is determined by the relation

$$
\Sigma_{c r}=\frac{c^{2} D_{s}}{4 \pi G D_{d} D_{d s}} .
$$

In (4), $c$ is the speed of light, $G$ the gravitational constant, $D_{s}$ the distance between the observer and lensed source, $D_{d}$ the distance between the observer and lens, and $D_{d s}$ the distance between the lens and source.

Let us adopt the radius of the cluster $R_{x}$ as a scaling parameter $\xi_{0}$ and introduce dimensionless vectors in the plane of the lens $\mathbf{x}=\xi / \xi_{0}$ and the plane of the source $\mathbf{y}=\eta / \eta_{0}$, where $\eta_{0}=\xi_{0} D_{s} / D_{d}, \xi_{0}=R_{x}, \mathbf{x}\left(x_{1}, x_{2}\right)$, and $\mathbf{y}\left(y_{1}, y_{2}\right)$. We use $\alpha(\mathbf{x})$ to denote the normalized vector $\alpha^{0}\left(\xi_{0} \mathbf{x}\right)$ :

$$
\alpha(\mathbf{x})=\frac{D_{d} D_{d s}}{\xi_{0} D_{s}} \alpha^{0}\left(\xi_{0} \mathbf{x}\right) .
$$

The gravitational lens equation (Kochanek, Schneider, \& Wambsganss 2006; Zakharov 1997) can then be written in the form

$$
\mathbf{y}=\mathbf{x}-\alpha(\mathbf{x}) .
$$

The possibility of WIMP clusters being oblate by rotation can hardly provoke any fundamental objections. However, the questions of the oblate cluster's shape and density distribution have no simple answers. The branch of celestial mechanics that studies the equilibrium shapes of rotating bodies assumes them to be comprised of a viscous incompressible fluid, which is clearly inappropriate for systems of weakly interacting particles. Nonetheless, some conclusions may be of interest for our case as well. Given the presence of a small dense core and a rapid decrease of the particle density (1) with distance from the cluster center, we can describe this density distribution to a first approximation using the Roche model, which assumes that the entire mass of the rotating body is concentrated at its center (Subbotin 1949). This type of model has been used earlier to study planetary atmospheres and protoplanetary disks. The oblateness of the marginally stable surface of the Roche model was fairly small, with a polar-to-equatorial-axis ratio equal to $2 / 3$ (Subbotin 1949).

Models with a nonsingular power-law elliptical distribution of the surface mass density have been widely used in studies of transparent gravitational lenses:

$$
\kappa(\mathbf{x})=\frac{q}{\left(u^{2}+x_{1}^{2}+x_{2}^{2} / k^{2}\right)^{n}} .
$$

This model has a constant-density core of radius $u$ at the center. The contours of equal surface density are ellipses with fixed minor to major semiaxis ratio $k=b / a$. Models of the form (6) are believed to adequately describe the surface mass density distributions in galaxies for a wide range of degrees of oblateness.

We first analyzed the case of a non-rotating cluster and tried to fit the density distribution of a WIMP cluster (3) with $r_{c}=0.07 R_{x}$ and normalization $\kappa(\mathbf{0})=1$ using the model (6) with $k=1$, choosing model parameters to minimize the sum of squared residuals. The solution of the corresponding optimization problem yielded the best-fit parameter values $q=1.04, u=0.09$, and $n=0.68$. The surface density distribution for model (6) with these parameters is a good fit to the initial distribution. We then assumed that, as the particle cluster rotates, the parameters $q, u$, and $n$ remain constant and the ellipses of equal density experience oblateness equal to that of the marginally stable surface in the Roche model: $k=2 / 3$.

It is well known that a transparent gravitational lens with surface density distribution (6) can have caustics in the source plane, which, when crossed by a lensed star, can produce a large variety of light curve shapes. For this to happen, it is sufficient for a point $\mathbf{x}$ with $\kappa(\mathbf{x})>1$ to exist in the plane of the lens (Kochanek, Schneider, \& Wambsganss 2006; Zakharov 1997). This condition can be satisfied by the WIMP clusters considered. Let us fix $R_{x}=10^{14} \mathrm{~cm}$ and $M_{x}=0.5 M_{\odot}=10^{33}$ g. It then follows from (1) that $\rho_{0}=1.8 \times 10^{-8} \mathrm{~g} / \mathrm{cm}^{3}$, and the computation of integral (2) yields a surface density of $\Sigma(\mathbf{0})=3.4 \times 10^{5} \mathrm{~g} / \mathrm{cm}^{2}$ at the lens center. We find from (4) that the critical density is $\Sigma_{c r}=5.3 \times 10^{4} \mathrm{~g} / \mathrm{cm}^{2}$ and $\Sigma_{c r}=1.7 \times 10^{5} \mathrm{~g} / \mathrm{cm}^{2}$ for 


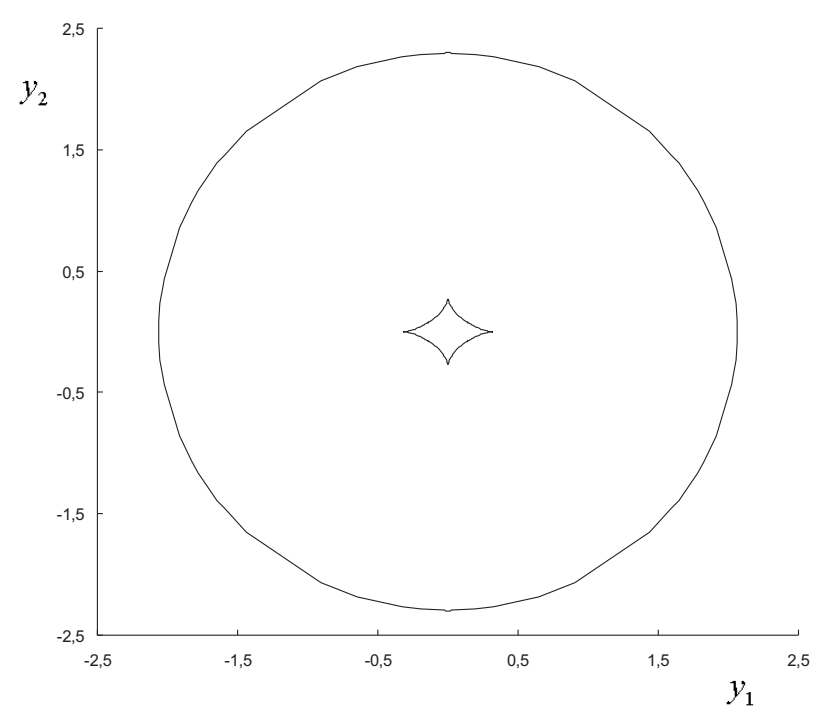

Fig. 1 Caustic curves of the non-compact WIMP lens in the plane of the source

typical cases of lensing of stars in the Large Magellanic Cloud by an object located in the Galactic halo $\left(D_{d}=8\right.$ $\mathrm{kpc}, D_{s}=46 \mathrm{kpc}$, and $\left.D_{d s}=38 \mathrm{kpc}\right)$ and lensing of Galactic bulge stars $\left(D_{d}=4 \mathrm{kpc}, D_{s}=8 \mathrm{kpc}\right.$, and $D_{d s}=4 \mathrm{kpc}$ ), respectively. Thus, the required excess of the density over its critical level is quite possible.

Let us assume for the sake of simplicity that the rotational axis of the cluster lies in the plane of the lens and that the major axes of the ellipses of equal surface density form intervals on the $x_{1}$ axis. It is necessary however to note that this alignment of cluster maximizes the asymmetry of the lens and the size of the caustics. Barkana (1998) has developed an efficient algorithm for solving the gravitational-lens equation (5) using the density distribution (6) and written a FORTRAN code implementing this algorithm. For a given vector $\mathbf{x}$, the code computes the components of the deflection angle vector $\alpha(\mathbf{x})$ and the elements of the Jacobi matrix $A_{i, j}=\partial y_{i} / \partial x_{j}$. Our computations for model (6) with the parameters adopted here demonstrated the existence of critical curves in the plane of the lens, defined by the condition $\operatorname{det} A=0$. The corresponding caustics in the plane of the source are shown by the solid curves in Figure 1. When a point source crosses the caustic, the gravitational lens amplification factor $\mu=1 /|\operatorname{det} A|$ can formally approach infinity. For a source with a finite angular size, $\mu$ is finite but can achieve rather large values.

For the adopted size of the WIMP cluster, the unit scale length in the plane of the lens for $D_{d}=4 \mathrm{kpc}$ corresponds to an angle of $0^{\prime \prime} .0017$, which is much smaller than the resolution of current telescopes. As a result, the object should show up as a microlens, with the total flux from all its images being observed. Due to the orbital motion of the observer, particle cluster, and lensed star, the observed flux should vary with time. Exact computation of the light curves is computer intensive. However, it is easy to obtain a qualitative description of the observational picture. When a source of finite angular size crosses the outer caustic, two additional source images should appear and the observed flux should increase. However, the flux amplification factor on this caustic is comparatively small, and the caustic crossing could escape detection. When the source crosses the inner caustic, two more source images appear, resulting in a sharp increase of the flux followed by a slower decrease. At the second crossing of the inner caustic, the two images merge and disappear, inverting the pattern of the flux variations. When the line of the relative motion of the lensed star in Fig. 1 passes far from the cusp and is parallel to the coordinate axes when it crosses the inner caustic, the light curves have symmetric profiles with two maxima and a dip at the center. Such curves can be realized in the noncompact microlens model analyzed by Zakharov (1999). If the star moves along a line at some angle to the $y_{1}$ axis, either the first or second crossing of the inner caustic should take place closer to the corresponding cusp. As a result of the difference in the amplification factors, the light curve becomes asymmetric and either its first or second maximum becomes stronger. Similar light-curve shapes can appear in the cases of binary gravitational lenses and have been observed in actual microlensing events. We must therefore bear in mind the possibility that at least some such events could in reality be associated with WIMP clusters. When the star goes beyond the outer caustic, comparative modest flux variations will again be observed as a flux increase followed by a more abrupt decrease. If the lensed star does not cross the outer caustic, the flux-variation curve is symmetrical and has a single maximum.

The microlensing pattern considered above depends on a large number of parameters. Obviously, it is possible to obtain a large variety of light curves by changing the lens orientation and varying the parameters for the density distribution and the relative motion of the lensed star. Represents the interest to examine quantitative a question how well a light curve for binary lens can be fitted by curve for the model of noncompact lens.

\section{Spatial - Temporal Tunnels as Gravitational Microlenses}

As was shown by Morris \& Thorne (1988), Hochberg \& Visser (1997), and Hochberg, Popov, \& Sushkov 
(1997), the equations of general relativity allow solutions in the form of two regions in space-time connected by a so-called spatial-temporal tunnel or natural wormhole. Such a tunnel can exist only if it is filled with a certain type of exotic matter with a negative energy density and, therefore, a negative mass. It was first suggested by Kim \& Cho (1996) that such an object could be manifest as a gravitational lens capable of forming multiple images of a distant source of radiation. Under certain conditions, the angular distance between the images can become smaller than the resolving power of telescopes, and the observer will detect only the combined flux from all the images of the source. In this way, the wormhole will be manifest as a gravitational microlens with negative mass. Such a microlens modeled as a negative point mass was considered by Eiroa, Romero, \& Torres (2001), and Safonova, Torres, \& Romero (2002) using the commonly applied approximations of geometric optics and small deflection angles. Like an ordinary gravitational lens, it is basically specified by the angular radius of the Einstein cone $p_{0}$, which can be expressed as

$$
p_{0}^{2}=\frac{4 G|M|}{c^{2}} \frac{D_{d s}}{\left(D_{d s}+D_{d}\right) D_{d}},
$$

where $M$ the mass of the lens. Unfortunately, any reliable estimations of mass of wormholes and hence $p_{0}$ until today are absent.

The lens equation indicates that its properties differ dramatically from those of an ordinary positive- mass Schwarzschild lens due to the presence of a circular caustic with angular radius $2 p_{0}$ in the source plane. When a point source is located an angular distance $p<2 p_{0}$ from the lens, it is no longer observed, and the detected flux decreases to zero. When $p>2 p_{0}$, two images of the source arise, located on one side of the lens between the directions towards the lens and the source. One of the images is always inside the Einstein cone, while the second is outside it. As the source approaches the caustic from the outer side, the images merge and disappear when $p=2 p_{0}$. The amplification factor for the flux from a source with an infinitely small angular size in the region $p>2 p_{0}$ is

$$
A(u)=\frac{u^{2}-2}{u \sqrt{u^{2}-4}},
$$

where $u=p / p_{0}$; at a caustic crossing, $A(u)$ formally tends to infinity. Relation (8) differs from the corresponding formula for a Schwarzschild lens (Kochanek, Schneider, \& Wambsganss 2006; Zakharov 1997) only in the signs of the numerical terms in the numerator and denominator.
In the case of lensing of a source with a finite angular size, the flux will increase as it approaches the caustic, then sharply decrease and completely disappear. In the course of the egress of the source from the inner region of the circular caustic, the pattern will change to the opposite. For a source with a circularly symmetrical brightness distribution, the light curve will always be symmetrical relative to the time of its closest approach to the lens. If the source does not cross the caustic during its relative motion, the light curve displays one maximum and its shape is close to those of observed stellar microlensing light curves. As it was shown by Bogdanov \& Cherepashchuk (2002), if a point source moves outside the circular caustic, photometric observations cannot distinguish light curves due to a wormhole and a positive-mass gravitational lens. However, it is apparent that the amplification factors of the lenses can be equal only for one value of the impact parameter, so that the light curves for extended sources will differ.

Images and light curves of extended sources formed by a negative-mass lens were calculated by Safonova, Torres, \& Romero (2002) using the method of ray tracing and the solution of the lens equation on a $5000 \times 5000$ count grid. If we consider only photometric effects, the problem is simplified by the fact that the amplification factor for the flux from a source area element will be specified by (8). The problem is thus similar to the calculation of light curves for the case when a source is lensed by a linear caustic (Bogdanov \& Cherepashchuk 2000). In this case, photometric effects depend only on the one-dimensional projection of the source brightness distribution onto the axis perpendicular to the caustic usually called the strip brightness distribution. In our case, the lens possesses a circular caustic, and the light curve $I(p)$ as a function of the angular distance from the lens $p$ will depend on the onedimensional brightness distribution $B_{p}(r)$, which is the source brightness integrated over an infinitely narrow circular strip with radius $r$ concentric to the caustic. Within this strip, the lens amplification factor (8) remains constant. If the angular size of the source is much smaller than $p_{0}$, the curvature of the caustic can be neglected, and the distribution $B_{p}(r)$ coincides with the strip brightness distribution. As in the previous case of a linear caustic, the influence of the singularity of the integral in the flux calculation can be removed by corresponding selection of the grids for the variables $p_{i}$ and $r_{i}$ (Bogdanov \& Cherepashchuk 2002).

Further, we will assume the lensed source to be a star and the brightness distribution over the stellar disk to display circular symmetry. In this case, the detected flux will depend only on the angular distance between 
the center of the stellar disk and the lens. For most stars, the limb darkening is described by the linear law:

$$
b_{\lambda}(\mu)=b_{\lambda}(1)\left(1-x_{\lambda}+x_{\lambda} \mu\right)
$$

where $\mu$ is the cosine of the angle between the line of sight and the normal to the stellar surface and $x_{\lambda}$ is the limb-darkening coefficient, which depends on the wavelength $\lambda$. The value $\mu$ corresponding to the angular distance from the center of the star $\rho$ is related to it by the expression:

$$
\mu=\sqrt{R^{2}-\rho^{2}} / \rho,
$$

where $R$ is the angular radius of the star. Further, we will assume that the brightness at the center of the star can be written

$$
b_{\lambda}(1)=1 / \pi R^{2}\left(1-x_{\lambda} / 3\right),
$$

which normalizes its flux to unity.

Let us introduce a polar coordinate system $(r, \varphi)$ with its origin coincident with the lens. If the center of the stellar disk is located an angular distance $p$ from the lens, the integrated brightness of the circular strip of the disk specified by the radius $r$ is

$$
B_{p}(r)=2 \int_{0}^{\varphi_{m}} b_{\lambda}(\rho) r d \varphi,
$$

where $\rho^{2}=r^{2}+p^{2}-2 r p \cos \varphi$ and an upper limit for the integral can be derived from the condition $R^{2}=$ $r^{2}+p^{2}-2 r p \cos \varphi_{m}$. Taking into account the caustic amplification factor (8), the detected radiation flux is

$$
I(p)=\int_{p-R}^{p+R} A\left(r / p_{0}\right) B_{p}(r) d r,
$$

where $A\left(r / p_{0}\right)=0$ for $r<2 p_{0}$.

For the purpose of the estimation of the effect of finite angular size of the source we chose a $K 0 \mathrm{~V}$ star, and assumed that its limb-darkening coefficient is $x_{V}=$ 0.702 in the $V$ band (Rubashevskii 1990). The light curves were calculated using (9), (10) and (11) and canonical-dissection grids with a step sufficiently small that the relative error would not exceed $10^{-4}$. Since the flux depends only on the angular distance $p$, the light curve can be calculated for a single value of the impact parameter $p_{m}=0$; we can find the counts for other values via interpolation. We used a cubic spline interpolation, which provided the necessary accuracy. We chose the value $R=0.10$ for the comparison with the calculations of Eiroa, Romero, \& Torres (2001) (further, we will measure all angles in units of $p_{0}$ ).

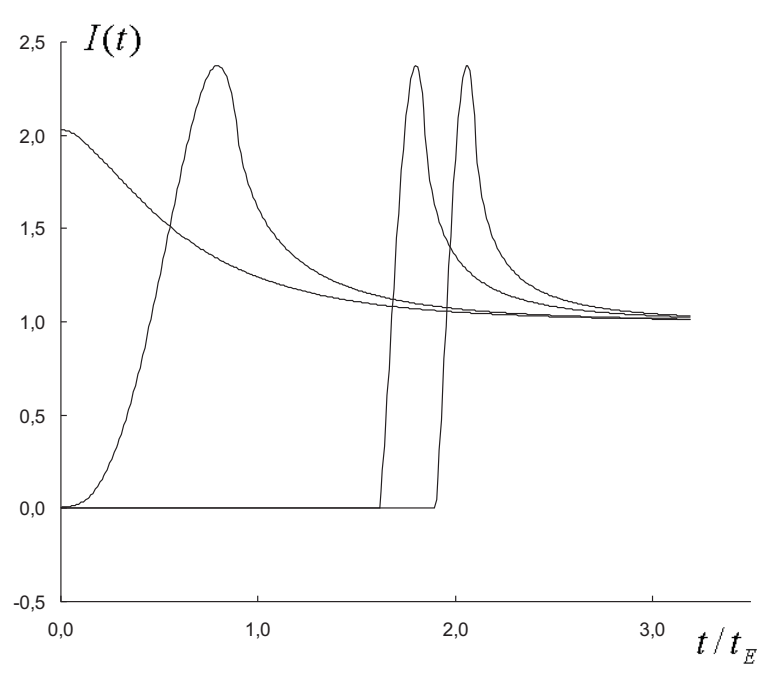

Fig. 2 V light curves for the microlensing of a star with angular radius 0.10 by a wormhole; time is measured in units of the Einstein cone angular radius crossing time. The positive branches of the curves for the impact parameters $p_{m}=2.1,1.9,1.0$, and 0 are indicated

The light curves were calculated for the impact parameters $p_{m}=0,1.0,1.9$, and 2.1. Further, we will measure time in units of the time required to cross the angular radius of the Einstein cone $t_{E}$. Figure 2 presents these results. Since all the curves are symmetrical about the time of closest approach to the lens, the figure shows only their positive branches. Our calculations are consistent with the results of Eiroa, Romero, \& Torres (2001), confirming the validity of the technique used. We can see from Fig. 2 that, when the star does not cross the circular caustic, the light curve displays a single maximum and its shape is similar to that for a Schwarzschild lens.

When a star crosses the circular caustic, characteristic symmetrical light curves with a central depression are formed. They differ markedly from observed stellar microlensing light curves, and could potentially provide unambiguous evidence for the presence of a negativemass gravitational lens. However, in the study by Bozza et al. (2002) of microlensing of stars by a compact object surrounded by an extended gaseous envelope, due to the extinction of the stellar radiation by refraction and Rayleigh scattering in the envelope, the light curve can acquire a shape close to that characteristic of a negative-mass lens. One possibility for discriminating between these two types of lens is provided by analyses of chromatic effects associated with the lensing.

The basic property of gravitational lenses of both positive and negative masses is that their parameters are independent of wavelength. However, the amplification factors are different for radiation from different 
regions of the stellar disk. Due to the wavelength dependence of the brightness distribution, this results in chromatic effects during the microlensing. The possibility of observing these effects for ordinary gravitational lenses has been considered, for example, in papers of Bogdanov \& Cherepashchuk (1995), Loeb \& Sasselov (1995), and Han, Park. \& Jeong (2000). Such an analysis for a negative-mass lens was carried out in paper of Eiroa, Romero, \& Torres (2001) by calculating the $U$ and $I$ light curves for a $K$ giant with $T_{\text {eff }}=4750 \mathrm{~K}$ and $R=0.10$, assuming that the limb-darkening coefficients were $x_{U}=1.050$ and $x_{I}=0.503$. The limb darkening coefficients $x_{\lambda}$ were determined from calculations of stellar atmosphere models. In this case, $x_{\lambda}$ can be estimated in different ways, based on either conservation of the total flux or the best consistency between (9) and the calculated data (Rubashevskii 1990). The first method can formally give a value $x_{\lambda}>1$. However, it is physically meaningless to use this value to calculate the brightness distribution (9), as was done in paper of Eiroa, Romero, \& Torres (2001), since this yields negative brightness at the edge of the stellar disk.

We calculated the variations of $V-I$ for microlensing of a $K 0 \mathrm{~V}$ star by a negative-mass object, since exactly these photometric bands are most frequently used in searches for gravitational- microlensing events. The limb-darkening coefficients $x_{V}=0.702$ and $x_{I}=0.433$ were taken from paper of Rubashevskii (1990). As for the calculation of the photometric effects, we adopted $R=0.10$ and $p_{m}=0,1.0,1.9,2.1$. Figure 3 presents the resulting positive branches of the color-variation curves. As expected, the maximum variation $\Delta(V-I)$, which corresponds to reddening, occurs when the stellar disk is almost entirely inside the circular caustic. However, the radiation flux is very small in this case (see Fig. 2) and cannot be detected. We restricted the calculations to the region in which the $V$ flux exceeds 0.001 of its initial value. In the course of the passage of the stellar disk across the caustic, $\Delta(V-I)$ changes sign twice: after reddening, the star becomes bluer, then redder again. However, in the region where the flux is appreciably different from zero, the chromatic effects are small and can be detected only with rather high photometric accuracy. If the star does not cross the circular caustic, the color varies in the same way as for a Schwarzschild lens (Bogdanov \& Cherepashchuk 1995) and reddening is always observed.

Thus, in the same way as for ordinary gravitational lenses, chromatic effects during the microlensing of a star by a wormhole are second-order compared to photometric effects. However, their detection could make it easy to discriminate between this exotic object and a compact body with an extended gaseous envelope. In

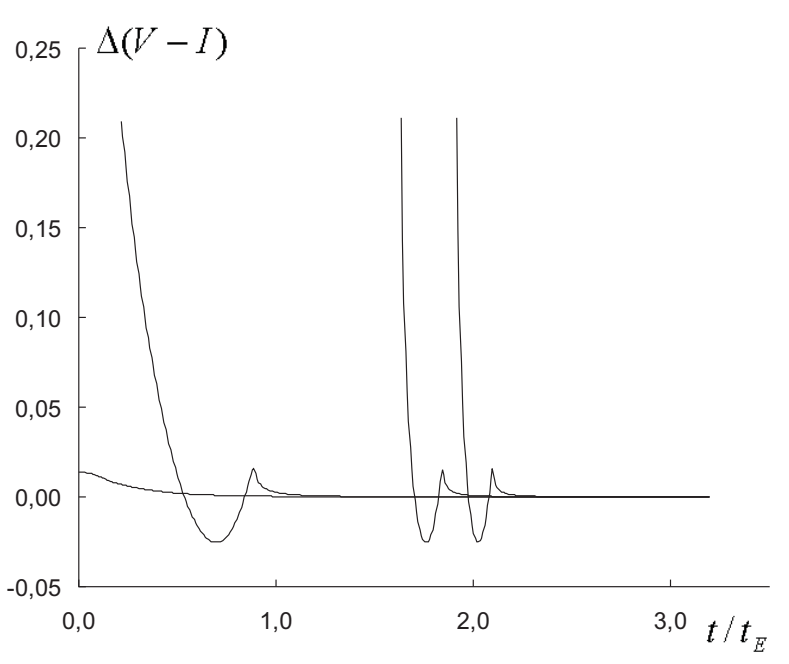

Fig. 3 Variation of the color index $\Delta(V-I)$ for the microlensing of a star by the wormhole shown in Fig. 2 . The units along the horizontal axis are the same as in Fig. 2

the latter case, the chromatic effects will be very different in both their values and character, due to the strong wavelength dependence of the refraction and, especially, of Rayleigh scattering $\left(\propto \lambda^{-4}\right)$.

Analogous to chromatic effects, gravitational microlensing of stars gives rise to variable polarization of the observed radiation, due to the character of the polarization distribution over the stellar disk. The stellar radiation becomes polarized as a result of scattering in the stellar atmosphere. Chandrasekhar (1953) and Sobolev (1956) demonstrated that the radiation emerging from a plane-parallel Rayleigh atmosphere is partially polarized, with the direction of the electric vector being perpendicular to the plane containing the line of sight and the normal to the stellar surface. In ordinary observations of stars, the detected total radiation flux from the entire stellar disk is unpolarized due to the disk's symmetry. The origination of partial polarization of the total flux is associated with a violation of the disk's symmetry due to rapid rotation of the star, tidal deformation, the presence of hot spots and/or an asymmetrical envelope, or a combination of these factors. It is apparent that the action of a gravitational lens also violates the disk's symmetry. The type and degree of polarization observed during microlensing of a star by a Schwarzschild lens were considered in papers of Simmons. Willis, \& Newsam (1995) and Bogdanov, Cherepashchuk \& Sazhin (1996).

To analyze the linear polarization that originates during the propagation of radiation in the atmosphere of a star, it is convenient to introduce the intensities $b_{l}$ in the plane, which contains normal to the stellar 
surface and line of sight, and $b_{r}$ in the direction perpendicular to this plane. For simplicity, we will assume that the lensed star has a plane-parallel Rayleigh atmosphere. In this case, the exact brightness distributions $b_{l}$ and $b_{r}$ over the stellar disk are presented by Chandrasekhar (1953). For our estimates, it is more convenient to use the approximate formulas derived by Bochkarev \& Karitskaya (1983):

$$
\begin{array}{r}
b_{r}+b_{l}=\frac{1+16.035 \mu+25.503 \mu^{2}}{1+12.561 \mu+0.331 \mu^{2}}, \\
\frac{b_{r}-b_{l}}{1-\mu}=\frac{0.1171+3.3207 \mu+6.1522 \mu^{2}}{1+31.4160 \mu+74.0112 \mu^{2}},
\end{array}
$$

where, as in (9), $\mu$ is the cosine of the angle between the line of sight and the normal to the stellar surface. Relations (12) and (13) can be used to estimate the intensities of the polarized components, so that the relative error of the degree of polarization $P=\left(b_{r}-b_{l}\right) /\left(b_{r}+b_{l}\right)$ compared to the exact solution for any $\mu$ does not exceed 0.001 .

Let us bring the origin of the polar coordinate system $(\rho, \theta)$ to the center of the stellar disk, so that the main axis from which we measure the angle $\theta$ will be directed towards the gravitational lens, located an angular distance $p$ from the coordinate origin. Due to the circular symmetry of the initial brightness distributions $b_{l}$ and $b_{r}$, it is apparent that the flux and degree of polarization of the detected radiation will depend only on $p$. Let $b_{L}(\rho, \theta)$ and $b_{R}(\rho, \theta)$ be the intensities at the point of the visible stellar disk with the coordinates $(\rho, \theta)$ for observations through polarizers oriented parallel and perpendicular to the direction towards the lens. Then, according to the transformation law for the Stokes parameters (and, accordingly, for the intensities $b_{l}$ and $b_{r}$ ), we have:

$$
\begin{aligned}
& b_{L}(\rho, \theta)=b_{r}(\rho) \sin ^{2} \theta+b_{l}(\rho) \cos ^{2} \theta, \\
& b_{R}(\rho, \theta)=b_{l}(\rho) \sin ^{2} \theta+b_{r}(\rho) \cos ^{2} \theta .
\end{aligned}
$$

The coordinate system $(\rho, \theta)$ is related to the system $(r, \varphi)$ introduced above: $r \sin \varphi=\rho \sin \theta$. Taking this into account and substituting the brightness distributions (14) and (15) into (10) and (11), we can calculate the flux from the star with the polarizer oriented in the parallel $\left(I_{L}(p)\right)$ and perpendicular $\left(I_{R}(p)\right)$ directions relative to the lens. The degree of polarization $P(p)$ when the center of the star is an angular distance $p$ from the lens will be

$$
P(p)=\frac{I_{R}(p)-I_{L}(p)}{I_{R}(p)+I_{L}(p)} .
$$

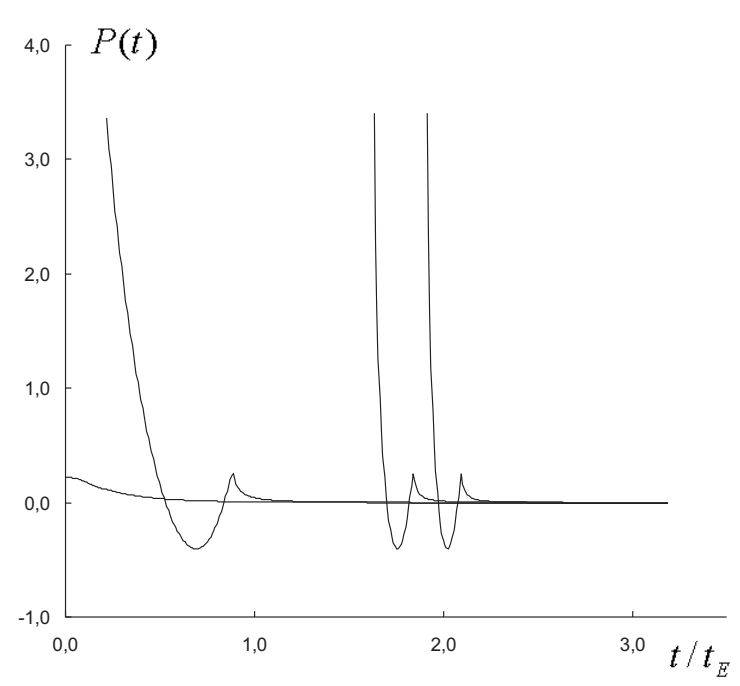

Fig. 4 The variation of the degree of polarization $P(t)$ (in percent) for curves of microlensing of a star by the wormhole shown in Fig. 2. The units along the horizontal axis are the same as in Fig.2

Figure 4 presents the positive branches of the polarization curves for the microlensing of a star with a Rayleigh atmosphere calculated for the same lensing parameters as in the previous case. As previously, we will consider only those sections of the curves for which the flux is no lower than 0.001 of the initial value. Since, like the chromatic effects, the polarization effects of microlensing are due to differences in the amplification of the radiation from the central and limb regions of the stellar disk, it is not surprising that the shape of the polarization curves essentially coincides with that of the color-index variation curves (see Fig. 3). During the passage of the caustic across the stellar disk, the degree of polarization changes sign twice. According to (16), this means that the plane of the electric vector is first perpendicular $(P>0)$, then parallel $(P<0)$, and finally perpendicular $(P>0)$ to the direction towards the lens.

When the star does not cross the circular caustic, the degree of polarization is always positive, and the plane of the electric vector is always perpendicular to the direction from the center of the star towards the gravitational lens. Thus, with sufficient observational accuracy, it may be possible to detect the rotation of the plane of polarization as the star moves relative to the lens. At the time when the maximum degree of polarization is attained, which coincides with the maximum of the flux from the lensed star, the plane of polarization will be specified by the position angle of the relative motion of the star and lens. The direction of the motion remains unspecified, due to the symmetry. 
A similar pattern is observed for a Schwarzschild lens (Bogdanov, Cherepashchuk \& Sazhin 1996).

For a star with a pure Rayleigh atmosphere, the degree of polarization is small, and its maximum, reached at the very edge of the stellar disk, is $11.7 \%$. Since the albedo of a singular scattering under the conditions in stellar atmospheres is always smaller than unity, we expect that the polarizations of real stars will be lower, so that the calculated values correspond to upper limits. In addition, the Nagirner effect may also be observed (Nagirner 1962), which is manifest by a rotation of the plane of polarization with motion from the edge of the stellar disk. The influence of the true absorption on the polarization of the radiation emerging from the atmosphere of the star assuming a plane-parallel geometry was considered in detail by Bochkarev, Karitskaya, \& Sakhibullin (1985). In the Wien region of the spectrum, $P$ can appreciably exceed the value for a Rayleigh atmosphere. The degree of polarization for long wavelengths is smaller, but remains appreciable. For latetype dwarfs, which statistically are most likely to be lensed, an additional contribution to the increase of $P$ may be introduced by Rayleigh scattering on molecules. Overall, for real stars, the order of magnitude of the calculated polarizations is expected to remain valid.

Thus, both chromatic and polarization effects during the microlensing of a star by a wormhole are small. However, the accuracy of current polarimetric observations considerably exceeds photometric accuracy; for sufficiently bright objects, $P$ can be determined with an error not exceeding $0.01 \%$. Unfortunately, this accuracy cannot be provided in monitoring observations during searches for microlensing events. However, observers have already accumulated considerable experience in triggering large telescopes to observe detected events involving microlensing by gravitationallens caustics, so that prospects for observing these polarization effects are fairly encouraging.

\section{Microlensing of Stars by NUT Gravimagnetic Lenses}

Newman, Unti, and Tamburino (NUT, 1963) considered a space-time metric that is a generalization of the Schwarzschild metric. Along with the mass of ordinary matter, $M$, it is also determined by the so-called NUT factor, $l$, which describes the contribution of magnetic monopole to the curvature of space-time. The parameter $l$ is often simply called the magnetic mass. The NUT metric becomes the Schwarzschild metric for $l \rightarrow 0$. On the other hand, gravitation still exists as $M \rightarrow 0$ (the "pure NUT" case).
The ability of the NUT objects to be gravitational lenses was studied by Nouri-Zonoz \& Lynden-Bell (1997). Generally, the properties of such lenses are close to those of Schwarzschild lenses. Under certain conditions, they are also capable of forming multiple images of lensed objects and amplifying the light flux detected by the observer. However, there are two important differences: the presence of a round zone where the lensed source is invisible, with its center towards the lens and its size increasing with the NUT factor, $l$; and a shear of the source images, manifests as a rotation in the plane of the sky around the optical axis of the lens.

It is possible that the angular separation between the source images formed by a NUT lens will be smaller than a telescope's resolution. In this case, we are dealing with gravitational microlensing, and the observer will detect only variations of the light flux during the lens's motion relative to the source. This situation was analyzed for NUT lensing of a point source in papers of Rahvar \& Nouri-Zonoz (2003) and Rahvar \& Habibi (2004), where the results were also compared to lensing by a Schwarzschild lens.

The amplification coefficient for the flux from a point source lensed by a Schwarzschild lens is given by (Kochanek, Schneider, \& Wambsganss 2006; Zakharov 1997)

$$
A(u)=\frac{u^{2}+2}{u \sqrt{u^{2}+4}} .
$$

Here as earlier, $u=p / p_{0}$ is the angular distance between the lens and the source measured in Einstein cone angular radii. When describing microlensing by a NUT object, a new parameter is introduced:

$$
N=\frac{1}{p_{0}} \sqrt{\frac{2 l D_{d s}}{D_{d} D_{s}}} .
$$

When $u^{2}<2\left(\sqrt{4 N^{4}+1}-1\right)$, the amplification coefficient of a NUT lens is imaginary, corresponding to an absence of source images. Outside this region, the amplification coefficient for the flux from an infinitely small source is given by Rahvar \& Nouri-Zonoz (2003)

$$
\begin{gathered}
A(u)=\frac{1}{1-a_{-}}\left(1-\frac{8 N^{4}}{a_{-}^{-1}-1}\right)^{-1 / 2}- \\
\frac{1}{1-a_{+}}\left(1-\frac{8 N^{4}}{a_{+}^{-1}-1}\right)^{-1 / 2},
\end{gathered}
$$

where

$$
a_{ \pm}=\left[\frac{2+u^{2} \pm \sqrt{u^{4}+4 u^{2}-16 N^{4}}}{2\left(4 N^{4}+1\right)}\right]^{2}
$$

and the sign in the subscript of a corresponds to the sign in front of the square root. An approximate expression 
for the amplification coefficient valid for small $N$ and $A(u)$ is

$$
A(u)=\frac{u^{2}+2}{u \sqrt{u^{2}+4}}+\frac{8 N^{4}\left(u^{2}+2\right)}{u^{3}\left(u^{2}+4\right)^{3 / 2}} .
$$

Comparing (19) and (17), we note that amplification by a NUT lens is stronger than amplification by a Schwarzschild lens when $u^{2}>2\left(\sqrt{4 N^{4}+1}-1\right)$. With decreasing magnetic mass $(N \rightarrow 0)$, the second term in (19) vanishes, and this equation becomes equal to (17). The presence of the source invisibility zone for $N$ not equal to zero limits the minimum value of $u$, and decreases the maximum possible amplification coefficient for a NUT lens, compared to a Schwarzschild lens.

In a microlensing event for a real star with finite angular size, the flux from each infinitesimal area of its disk will be multiplied by the amplification coefficient (17) or (19), depending on the lens type. Let us assume for simplicity that the brightness distribution across the stellar disk, $b_{\lambda}$, which depends on the wavelength $\lambda$, can be represented with a linear law (9). Let $r$ be the stellar angular radius and $p$ the angular distance from the stellar disk center measured in Einstein angular radii $p_{0}$. The brightness distribution (9) can then be written

$$
b_{\lambda}(p)=b_{\lambda}^{0}\left(1-x_{\lambda}+x_{\lambda} \sqrt{r^{2}-p^{2}} / p\right),
$$

where $b_{\lambda}^{0}$ the brightness at the center of the stellar disk.

Searches for microlensing events are based on stellar photometry in a system close to the standard broadband $U B V R I$ system. We can write expressions similar to (9) and (20) for the brightness distributions across a stellar disk in these filters, introducing the brightness at the disk center and the limb-darkening coefficient for a given filter, for example, $b_{V}^{0}$ and $x_{V}$ for the $V$ filter. We obtain the flux in this filter in the absence of the lens, $H_{V}^{0}$, by integrating the brightness distribution over the entire visible stellar disk:

$$
H_{V}=\pi r^{2} b_{V}^{0}\left(1-x_{V} / 3\right) .
$$

The limb-darkening coefficients in the filters of the standard broadband system were computed by Rubashevskii (1990) for stars with various effective temperatures using Kurucz' grid of model atmospheres.

In the presence of a gravitational lens at an angular distance $u$ from the center of the stellar disk, the $V$ flux can be written

$$
\begin{gathered}
H_{V}(u)=\int_{0}^{2 \pi} d \varphi \int_{0}^{r} A\left(\sqrt{u^{2}+\xi^{2}-2 u \xi \cos \varphi}\right) \times \\
b_{V}^{0}\left(1-x_{V}+x_{V} \sqrt{r^{2}-\xi^{2}} / r\right) \xi d \xi,
\end{gathered}
$$

where the angle $\varphi$ is measured from the direction towards the lens and the amplification coefficient $A$ is given by (17) for a Schwarzschild lens or (18) for a NUT lens. Similar expressions can also be written for the fluxes in other filters. Taking into account the dependence of $u$ on time, $t$,

$$
u(t)=\sqrt{u_{0}^{2}+\frac{\left(t-t_{0}\right)^{2}}{t_{E}^{2}}},
$$

where $u_{0}$ is the impact parameter - the minimum angular distance, achieved at the moment $t_{0}$, and $t_{E}$ is the time needed to cross the Einstein cone angular radius, we find the observed light curve, $I_{V}(t)$ :

$$
I_{V}(t)=H_{V}(t) / H_{V}^{0} .
$$

As where mentioned in section 3, the amplification coefficient for any gravitational lens is independent of wavelength. However, in the most probable case when the lens is not projected onto the stellar disk, the rapid decrease of $A(u)$ with increasing distance, $u$, leads to an increase in the fraction of light from the disk limb in the observed flux. For the $U B V R I$ filters, the limb darkening decreases with increasing maximum sensitivity wavelength. Thus, a chromatic effect, a kind of reddening, should be observed for real stellar microlensing events. This chromatic microlensing effect can be revealed by measuring color indices, for example,

$$
B-V=2.5 \lg \left(H_{V} / H_{B}\right)+C_{B-V}
$$

or

$$
V-R=2.5 \lg \left(H_{R} / H_{V}\right)+C_{V-R},
$$

with the constants $C_{B-V}$ and $C_{V-R}$ determined by the zero points of the photometric system. In the case of reddened star light, the value of the color indices will exceed the values in the absence of the lens, $(B-V)_{0}$ and $(V-R)_{0}$, and should be largest when the lensed star achieves its maximum light flux.

We computed the photometric and chromatic effects of microlensing a star by a NUT lens assuming, as in paper of Rahvar \& Nouri-Zonoz (2003) and Rahvar \& Habibi (2004), that $0 \leq N \leq 0.5$. We chose a main-sequence $K 0 \mathrm{~V}$ star with an effective temperature of $T_{\text {eff }}=4900 \mathrm{~K}$ and color indices $(B-V)_{0}=0.890$, $(V-R)_{\underline{\underline{n}}}=0.740$ (Allen 1973). According to Rubashevskii (1990), the limb-darkening coefficients for such a star derived from the condition of flux conservation are $x_{B}=0.859, x_{V}=0.702$, and $x_{R}=0.585$. We selected the brightnesses at the disk center so that the computed fluxes (21) in the absence of the lens would give the original color indices, $(B-V)_{0}$ and $(V-R)_{0}$. We assumed the most probable case of lensing, when the NUT- lens invisibility zone does not overlap with 


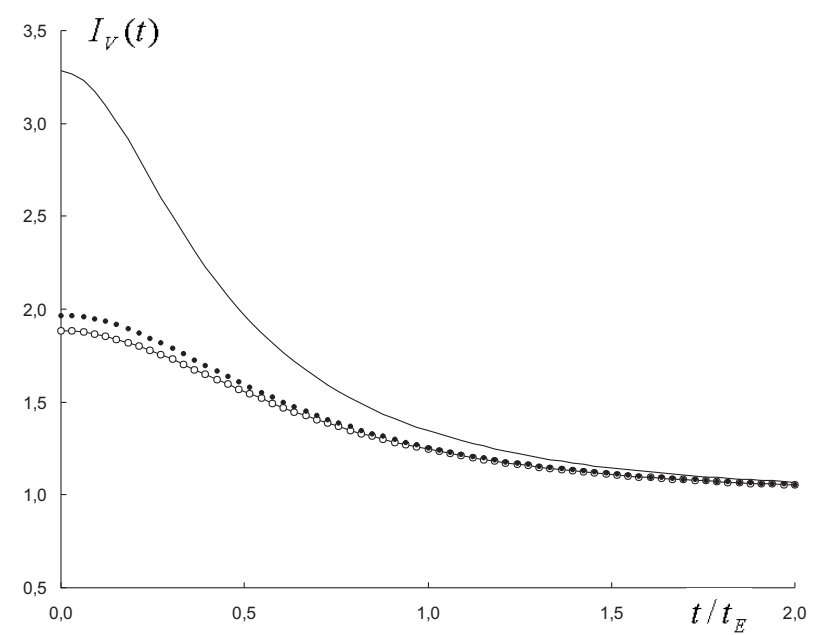

Fig. 5 Light curves for microlensing of a star with angular radius of $r=0.01$ by NUT lenses with $N=0.5$ (solid curve) and $N=0.3$ (dots), and by a Schwarzschild lens (circles)

the stellar disk. When $N=0.5$, the light from the source cannot be detected when $u<0.486$. We estimated the integrated fluxes (22) numerically with a relative uncertainty of $10^{-4}$.

Figure 5 displays the $V$ light curves for a star with $r=0.01$, barely different from a point source, with time measured in units of the Einstein cone angular radius crossing time, $t_{E}$, for $u_{0}=0.6$ and $t_{0}=0$. The curves are symmetrical about the maximum flux, and we show only their positive branches. The curve for $N=0.5$ (solid) is considerably different for the Schwarzschildlens curve, plotted as circles. In agreement with (19), the observed differences between the lenses quickly decrease with decreasing $N$. The light curve for $N=0.3$, plotted as dots, already displays little difference from the curve for a Schwarzschild lens, with the difference becoming virtually undetectable on the scale of the figure when $N=0.1$. No chromatic effects are observable for any $N$ values in the studied range, $0 \leq N \leq 0.5$. Deviations of the star's color indices from $(B-V)_{0}$ and $(V-R)_{0}$ are within the expected calculation uncertainties.

Figure 6 displays the results of our computations for a star with $r=0.10$, comparable to the Einstein cone angular radius, for $u_{0}=0.6$ and $t_{0}=0$. The results for lensing of the star by a NUT lens with $N=0.5$ is shown by the solid curve, while the dots show the light curve for a point source lensed by the same lens. The curve with circles is the light curve for lensing of the same star by a Schwarzschild lens, and virtually coincides with that for the point source on the scale of the figure. We can see that, in contrast to the Schwarzschild lens, the influence of the star's finite size is fairly appreciable in

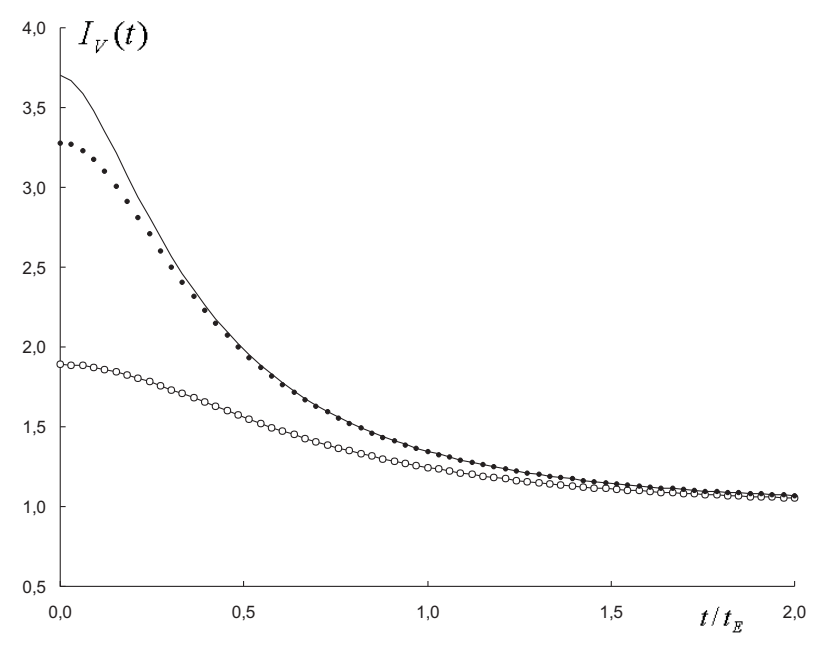

Fig. 6 Light curves for microlensing a point source (dots) and a star with angular radius $r=0.10$ (solid curve) by a NUT lens with $N=0.5$. Also shown is the light curve for microlensing of the same star by a Schwarzschild lens (circles)

the case of the NUT lens. Thus, the difference between the $I_{V}(t)$ curves for the two lens types becomes more significant as the star's angular size increases.

Figure 7 shows variations of the $B-V$ and $V-R$ color indices for the previous lensing case $(r=0.10$, $u_{0}=0.6$, and $\left.t_{0}=0\right)$. The solid curves show the results for the NUT lens $(N=0.5)$ and the dots the results for a Schwarzschild lens. Despite the smallness of the chromatic effect, it is significant for the NUT lens, whereas the star's color indices during lensing by the Schwarzschild lens are virtually undistinguishable from the initial values, $(B-V)_{0}$ and $(V-R)_{0}$. Obviously, the chromatic effect can be made stronger by a special choice of narrow observed spectral intervals. However, patrol stellar microlensing observations use broadband systems.

Another observable effect related to the predominant amplification of light from the disk limb can be manifest during stellar microlensing: linear polarization of the light can arise. The larger amplification coefficient of a NUT lens for fixed $u$ in the source visibility zone enhances this effect compared to a Schwarzschild lens. On the other hand, it causes a shear of the source images, rotating them in the plane of the sky; this should give rise to a rotation of the polarization plane, depolarizing the combined light flux. The simple approach to estimating this effect used in section 3 is not applicable here, and this problem must be resolved by analyzing the polarization properties of stellar images computed using ray tracing. 

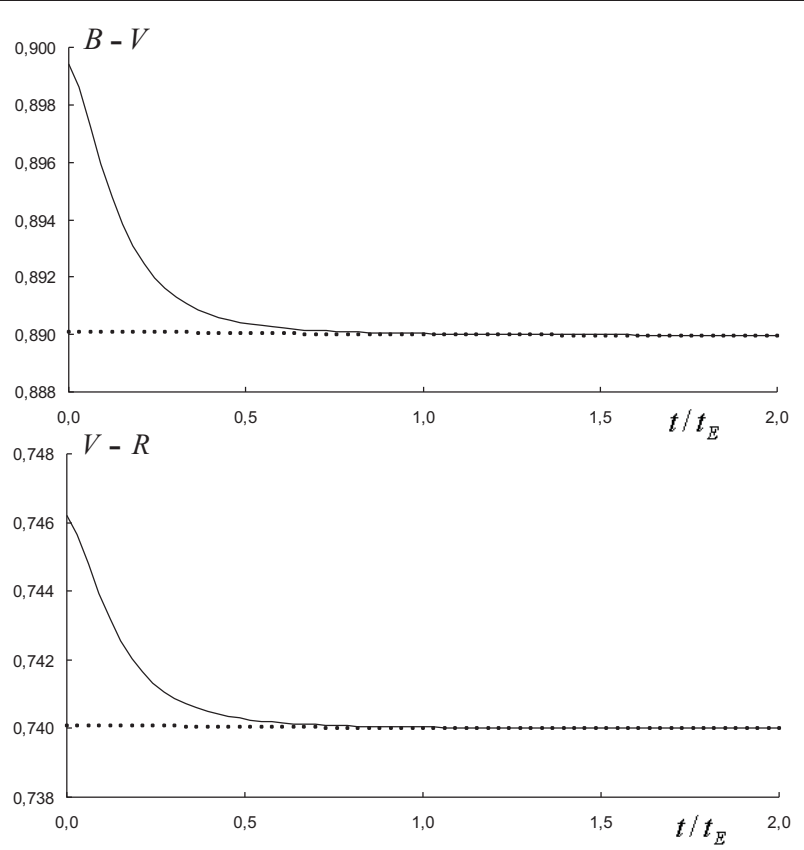

Fig. 7 Variations of the $B-V$ and $V-R$ color indices during microlensing of a star with angular radius $r=0.10$ by a NUT lens with $N=0.5$ (solid curve) and by a Schwarzschild lens (dots)

\section{Conclusion}

We assume that small-scale clusters of weakly interacting massive particles can, in the course of their evolution, acquire rotational momentum and spheroidal shape. Even a small degree of oblateness similar to that of the critical surface for a Roche model can result in the appearance of caustics in the plane of a lensed source. The multiple images that form cannot be resolved using current telescopes, and the cluster is manifest as a non- compact, spheroidal gravitational microlens. The crossing of caustics in the case of relative motion of the observer, particle cluster, and lensed star can produce a large variety of flux curves, including some observed in actual microlensing events that have been interpreted as manifestations of binary gravitational lenses. We must therefore bear in mind the possibility that at least some such events could in reality be associated with WIMP clusters.

The unambiguous identification of such an exotic object as a wormhole from observations of gravitational microlensing of stars is a rather complicated problem. If a source with a small angular size does not cross the circular caustic, it is impossible to discriminate between gravitational lenses with positive and negative mass. Even for stars with relatively large angular diameters, deviations from the light curve for a Schwarzschild lens are of the order of the observational errors. Distinc- tive photometric features of microlensing by a wormhole are a sharp decrease of the flux considerably below the initial level during the caustic crossing and a symmetric light curve. However, unavoidable gaps in the observations may prevent the detection of the second caustic crossing, and the shape of the light curve in the vicinity of the maximum is approximately the same as for microlensing by a caustic of an ordinary binary gravitational lens. When the angular size of the star is comparable to 0.10 of the Einstein cone radius for sufficiently large impact parameters, the observed light curves should resemble those for microlensing of a compact object surroundd by an extended gaseous envelope. This case can be easily distinguished from a negative-mass lens through an analysis of the chromatic and polarization effects arising during the lensing.

Our results demonstrate also that, during gravitational microlensing of stars, under certain conditions, the influence of the star's finite size and the brightness distribution across the stellar disk can facilitate the detection of lensing objects whose local space-time is described with the NUT metric. Such effects are virtually absent if the stellar angular radius is much smaller than the Einstein cone angular radius. If the size of the star becomes order 0.10 of the Einstein radius, lensing by a NUT lens exhibits considerable differences from lensing by a Schwarzschild lens, in terms of both flux amplification and color-index variations.

Acknowledgements This work was partially supported by the Russian Foundation for Basic Research, the Ministry of Science and Education of the Russian Federation, and the project "Universities of Russia". 


\section{References}

Alcock, C., Akerlof, C.W., Allsman, R.A. et al.: 1993, Nature $365,621$.

Alcock, C., Allsman, R.A., Alves, D.R. et al.: 2000, Astrophys. J. 542, 281.

Alcock, C., Allsman, R.A., Alves, D.R. et al.: 2001, Nature $414,617$.

Allen, C.W.: 1973, Astrophysical Quantities, 3rd ed. Athlone, London.

Barkana, R.: 1998, Astrophys. J. 502, 531.

Bennett, D.P., Becker, A.C., Quinn, J.L. et al.: 2002, Astrophys.J. 579, 639.

Bochkarev, N.G. \& Karitskaya, E.A.: 1983, Sov . Astron. Lett. 9, 6 .

Bochkarev, N.G., Karitskaya, E.A. \& Sakhibullin, N.A.: 1985,Astrophys. Space Sci. 108, 15.

Bogdanov, M.B.: 2001, Astron. Rep. 45, 929.

Bogdanov, M.B. \& Cherepashchuk, A.M.: 1995, Astron. Lett. 21, 505 .

Bogdanov, M.B., Cherepashchuk, A.M. \& Sazhin, M.V.: 1996, Astrophys. Space Sci. 235, 219.

Bogdanov, M.B. \& Cherepashchuk, A.M.: 1998, Astron. Rep. 42, 229.

Bogdanov, M.B. \& Cherepashchuk, A.M.: 2000, Astron. Rep. 44, 745.

Bogdanov, M.B. \& Cherepashchuk, A.M.: 2002, Astron. Rep. 46, 996.

Bozza, V., Jetzer, P., Mancini, L. \& Scarpetta, G.: 2002, Astron.Astrophys. 382, 6.

Byalko, A.V.: 1969, Sov. Astron. 13, 784.

Chandrasekhar, S.: 1953, Radiative Transfer, Dover Publ.

Eiroa, E., Romero, G.E. \& Torres, D.F.: 2001, Mod. Phys. Lett. A 16, 973.

Gaudi, B.S., Albrow, M.D., An, J.H. et al.: 2002, Astrophys. J.566, 463.

Gurevich, A.V. \& Zybin, K.P.: 1995, Phys. Lett. A 208, 276.

Gurevich, A.V., Zybin, K.P. \& Sirota, V.A.: 1997, Phys. Usp. 40, 869 .

Han, C., Park, S.-H. \& Jeong, J.H.: 2000, Mon. Not. R. Astron.Soc. 316, 97.

Hochberg, D. \& Visser, M.: 1997, Phys. Rev. D 56, 4745.

Hochberg, D., Popov, A. \& Sushkov, S.V.: 1997, Phys. Rev. Lett.78, 2050.

Hoyle, F.: 1949, in Problems of Cosmical Aerodynamics, Central Air Documents Office, Dayton, p. 195.

Kerins, E.J.: 1997, Astron. Astrophys. 322, 709.

Kim, S.-W. \& Cho, Y.M.: 1994, in Proceedings of the Seventh Marcel Grossman Meeting, Stanford University, 1994, World Scientific, River Edge, 1996, p. 1147.

Kochanek, C.S., Schneider, P. \& Wambsganss, J.: 2006, Gravitational lensing: strong, weak \& micro. Editors: G.Meylan,P.Jetzer, \& P.North, Springer-Verlag: Berlin.

Komberg, B.V., Kompaneets, D.A. \& Lukash, V.N.: 1995, Astron. Rep. 39, 402.

Kozlowski, S., Wozniak, P.R., Mao, S. \& Wood, A.: 2007, arXiv:astro-ph/0701488.

Loeb, A. \& Sasselov, D.: 1995, Astrophys. J. 449, L33.

Morris, M.S. \& Thorne, K.S.: 1988, Am. J. Phys. 56, 395.
Nagirner, D.I.: 1962, Tr. Astron. Obs. Leningr. Gos. Univ. 19, 79 (in Russian).

Newman, E.T., Tamburino, L. \& Unti, T.: 1963, J. Math. Phys. 4, 915.

Nouri-Zonoz, M. \& Lynden-Bell, D.: 1997, Mon. Not. R. Astron. Soc. 292, 714.

Paczynski, B.: 1986, Astrophys. J. 304, 1.

Peebles, P.J.E.: 1969, Astrophys. J. 155, 393.

Rahvar, S. \& Nouri-Zonoz, M.: 2003, Mon. Not. R. Astron. Soc. 338, 926.

Rahvar, S. \& Habibi, F.: 2004, Astrophys. J. 610, 673.

Rubashevskii, A.A.: 1990, Sov. Astron. 34, 433.

Safonova, M., Torres, D.F. \& Romero, G.E.: 2002, Phys. Rev. D 65, 023001.

Sazhin, M.V., Yagola, A.G. \& Yakubov, A.V.: 1996, Phys. Lett. A. 219, 199.

Simmons, J.E.L., Willis, J.P. \& Newsam, A.M.: 1995, Astron. Astrophys. 293, L46.

Sobolev, V.V.: 1956, Radiative Transfer in the Atmospheres of Stars and Planets Gostekhizdat, Moscow, (in Russian).

Subbotin, M.F.: 1949, Course of Celestial Mechanics. Vol.3, Gostekhizdat, Moscow, (in Russian).

Wozniak, P. \& Paczynski, B.: 1997, Astrophys. J. 487, 55.

Zakharov, A.F.: 1997, Gravitational Lenses and Microlenses, Yanus-K, Moscow, (in Russian).

Zakharov, A.F.: 1999, Astron. Rep. 43, 325.

This manuscript was prepared with the AAS $\mathrm{IAT}_{\mathrm{E}} \mathrm{X}$ macros v5.2. 